\title{
Same-age Tutoring in Maths using ICT: From Computational Math Students to Chemistry Students*
}

\author{
Ximo Gual-Arnau ${ }^{1}$ y Ana LLuch-Peris ${ }^{1}$ \\ ${ }^{1}$ Universitat Jaume I de Castelló
}

\begin{abstract}
In this work we present an educational project based on peer tutoring among students of the same age (same-age tutoring) who take the subject of Mathematics I but in two different degrees, such as the degree in Computational Mathematics and the degree in Chemistry. The contents of the subject are the same in both degrees, but both the students and the degrees themselves have completely different characteristics. Therefore, the students of the degree in Computational Mathematics will act as tutors and those of the degree in Chemistry as tutored students. In the tutoring sessions we use blended learning and ICT tools are introduced in a double sense; in order to manage communication, distribute teaching materials and carry out cooperative work, and with the aim to introduce a symbolic calculus program which helps to understand abstract mathematical concepts.
\end{abstract}

Keywords: same-age tutoring, ICT tools, Maths.

\begin{abstract}
Resumen
En este trabajo exponemos un proyecto educativo basado en la tutorización por pares entre estudiantado de la misma edad (same-age tutoring) que cursan la asignatura de Matemáticas I pero en dos grados diferentes como son el grado en Matemática Computacional y el grado en Química. Los contenidos de la asignatura son los mismos en los dos grados pero tanto el estudiantado como los propios grados tienen características completamente distintas. Por tanto, el estudiantado del grado en Matemática Computacional ejercerá de estudiantado tutor y el del grado en Química de estudiantado tutorizado. En las sesiones de tutorización utilizamos el aprendizaje semipresencial (blended learning) y se introducen las herramientas TIC en un doble sentido; con el fin de gestionar la comunicación, distribuir materiales docentes y realizar trabajo cooperativo, y con el objetivo de introducir un programa de cálculo simbólico que ayude a la comprensión de conceptos matemáticos abstractos.
\end{abstract}

Keywords: same-age tutoring, herramientas TIC, matemáticas.

${ }^{*}$ Proyecto financiado por la Universitat Jaume I de Castelló 


\section{Introducción}

La práctica mediante la cual existe una tutorización entre el estudiantado tiene una rica historia que se remonta a las civilizaciones clásicas; sin embargo, hasta estas últimas décadas la tutorización por pares no ha resultado de interés en la literatura científica, como una forma de mejorar el aprendizaje y beneficiar tanto al estudiantado tutor como al tutorizado (Robinson, Schofield y Steers-Wentzell 2005). Los efectos de la tutoría entre pares en Matemáticas se han documentado recientemente y los últimos estudios concluyen que las interacciones de los estudiantes durante la tutoría entre pares tienen efectos positivos significativos en el aprendizaje de matemáticas desde la educación preescolar hasta la educación superior (Alegre y col. 2020).

La tutoría entre pares (peer tutoring) se puede dar tanto entre estudiantado que pertenece a la misma edad (same-age tutoring) como entre pares donde estudiantado de mayor edad tutoriza al estudiantado más joven (cross-age tutoring). En este trabajo consideraremos una modalidad de tutoría entre pares de la misma edad pero donde estudiantado tutor y tutorizado pertenecen al primer curso de grados universitarios distintos. En concreto los tutores serán estudiantes del grado en Matemática Computacional y los tutorizados del grado en Química. Además, esta tutorización se realizará aprovechando las tecnologías TIC.

Como ya se apunta en Santaló (1986) existen dos hechos claros a la hora de abordar la enseñanza de las matemáticas: que las matemáticas no gustan a la mayoría de estudiantado de grados universitarios que no sean matemáticos y que las TIC han de introducirse en esta enseñanza.

Por tanto, aunque los temarios de las asignaturas de Matemáticas del primer semestre del primer curso de los grados de matemática computacional y química son muy parecidos, las características del estudiantado da cada grado y los objetivos de las asignaturas son muy distintos. Viene aquí, por tanto, a colación lo siguiente:

La profesora formula esta pregunta en la clase de matemáticas (Real-Pérez 2011):

- Si tuvierais que enseñar matemáticas a María... ¿qué deberíais saber primero?

- Matemáticas -responden a la pregunta de su profesora.

- No -corrige ella-, lo primero que debéis saber es quién es María.

De poco sirve saber muchas matemáticas, muchas teorías didácticas y muchas herramientas TIC, si no se tiene un conocimiento de las personas a las que tenemos que enseñar matemáticas, de sus intereses por el conocimiento de las mismas y del provecho que les han de sacar en función del grado universitario que cursen.

Por otro lado, las TIC pueden llegar a jugar un papel muy importante en el proceso de enseñanza y aprendizaje de las matemáticas, pero si se utilizan correctamente.

El uso de las TIC va a requerir que el profesorado tenga adquiridas una serie de competencias profesionales, no solamente en el uso de la herramienta que corresponda a cada momento, sino más importante todavía, en la metodología que va a utilizar y que será la que haga que el proceso alcance los objetivos que se hayan planteado inicialmente. Parte del grupo de herramientas TIC que se utilizan en la enseñanza de las matemáticas en los grados de Química y Matemática Computacional son iguales (aula virtual...). Sin embargo, el estudiantado de matemática computacional necesita conocer algunas herramientas y software específico, que no tiene porque conocer el estudiantado 
de químicas, pero sí le pueden ayudar a mejorar la comprensión de resultados matemáticos que ha estudiado. Una vez más, es muy importante conocer al estudiantado al que enseñamos matemáticas y los conocimientos y habilidades de qué dispone, los cuales podemos utilizar como recurso para facilitar el proceso de aprendizaje.

Las herramientas TIC son cada vez más habituales en las aulas universitarias. De hecho, en estos tiempos de pandemia derivada de la COVID-19, en muchos casos se han convertido en un elemento esencial para tratar de dar continuidad a esta nueva «anormalidad». En este trabajo pretendemos utilizar la innovación tecnológica como una herramienta ligada a la metodología docente same age-tutoring con la idea de alcanzar una innovación educativa, de forma que el estudiantado de Matemática Computacional, que dispone de habilidades sobre el funcionamiento de los ordenadores y la creación de contenidos digitales entendiendo mínimamente los lenguages de programación, utilice las herramientas digitales para el diseño de actividades didácticas activas en la tutorización a estudiantado de químicas, favoreciendo aprendizajes que van mucho más allá de las cuatro paredes del aula.

Por tanto, pretendemos que esta metodología complemente las clases magistrales que el profesorado utilizamos para transmitir información y los recursos que también el profesorado utilizamos para que el estudiantado consiga obtener las competencias genéricas y específicas de la asignatura de Matemáticas, de manera que la interacción entre estudiantado de diferentes grados, para los cuales las Matemáticas de primer curso y primer semestre, aún teniendo programas similares, presentan objetivos muy diferentes, consiga mejorar los resultados de aprendizaje del estudiantado de ambos grados.

\section{Objetivos}

En las primeras universidades de la antiguedad la figura del tutor designaba al maestro encargado de la tutela de un grupo de estudiantes sobre los cuales ejercía una especie de vigilancia educativa, con el fin de velar por su fidelidad a las verdades enseñadas. Posteriormente surge la tutoría integral como una alternativa a este concepto tradicional de tutoría, que se encuadra en el marco de un modelo formativo que pone en primer plano las interacciones profesor-estudiantes y de éstos entre sí, y propone construir otras formas de relación que vayan más allá del aula y de las consultas puntuales, de carácter instrumental. El rol del tutor también va cambiando hasta llegar a la tutoría entre iguales (peer-tutoring), una modalidad de aprendizaje cooperativo, en la que estudiantes más aventajados apoyan el aprendizaje de otros menos expertos, mediante un trabajo que se realiza en grupos pequeños o en parejas (Alzate-Medina y Peña-Borrero 2010).

Aunque en la mayoría de trabajos, a la hora de considerar la tutorización por pares entre estudiantado de la misma edad (same-age tutoring) se ha considerado que los y las estudiantes van a la misma clase, en este trabajo se ha considerado el same-age tutoring entre estudiantes del mismo curso pero de dos grados diferentes: el grado en Matemática Computacional y el grado en Química. Además, el rol del estudiantado será siempre el mismo: los tutores pertenecen al grado en Matemática Computacional y los tutorizados al grado en Química.

En el primer semestre de primer curso de los grados de Matemática Computacional y Química de la Universitat Jaume I de Castelló, ambos planes de estudio contemplan una asignatura denominada Matemáticas I, cuyos contenidos son álgebra lineal y cálculo diferencial. Sin embargo, se dan dos diferencias significativas que marcan la planificación y metodología didáctica empleada en la asignatura de cada uno de los grados. La primera es que al estudiantado de Matemática Compu- 
tacional le gustan las matemáticas, ha cursado con con éxito las matemáticas en el bachillerato científico-técnico y la nota de acceso a la titulación ha estado sobre los 11 puntos. Por su parte, al estudiantado del grado en Química no le gustan, en general, las matemáticas y la nota de acceso a la titulación ha estado sobre los 6.5 puntos. La segunda diferencia es el objetivo que se persigue en ambas asignaturas. En el grado de Matemática Computacional esta asignatura es un primer contacto con las matemáticas, que posteriormente se irá complementando con otras asignaturas del grado en cada uno de los cuatro cursos y lo que se pretende es que el estudiantado comprenda los conceptos básicos del álgebra y el cálculo diferencial y se empiece a familiarizar con distintos métodos de demostración. En el grado de Química esta asignatura es la única con contenidos de álgebra lineal y cálculo diferencial, y se pretende que el estudiantado entienda los conceptos y los aplique a problemas de carácter químico y físico.

Por otro lado, en lo que se refiere a las herramientas TIC, el estudiantado de los grados está familiarizado en el uso de herramientas básicas, en muchos casos a través de las aulas virtuales de las asignaturas, ya que condicionados por la COVID-19, la docencia de las asignaturas a lo largo del curso 2020/21 se ha realizado en modalidad semipresencial, siendo muchas clases de teoría, resolución de probleas y laboratorio en modalidad online. Sin embargo, el estudiantado de matemática computacional ha desarrollado y diseñado en otras asignaturas del grado que se impartían paralelamente a la de mátemáticas, programas de cálculo simbólico con determinados softwares informáticos, que se utilizan en la asignatura de matemáticas para visualizar mejor los contenidos de la misma. El uso de herramientas TIC, aunque no solucione ni de lejos los vacíos pedagógicos, si ayuda, y especielmente el el campo de las matemáticas, a una transformación pedagógica necesaria (Grisales-Aguirre 2018).

La propuesta metodológica consiste en realizar una tutoría entre pares, donde el papel del tutor o tutora recae en estudiantado del grado de Matemática Computacional y el papel de estudiantado tutorizado recae en el grado en Química. Con esta propuesta ambos salen beneficiados ya que al preparar y realizar la tutorización, el estudiantado de matemática computacional debe comprender los conceptos con detalle, visualizarlos a traves de herramientas TIC y ser capaz de transmitirlos a 'colegas' del grado en química. Por su parte, el estudiantado en química recibe los contenidos de una asignatura que, a priori no le gusta, a través de 'colegas' de matemática computacional.

Así pues, el proyecto combina las ventajas de los proyectos cross-age tutoring, donde el estudiantado tutor pertenece a cursos superiores y que, en general, son difíciles de planificar, y same-age tutoring, donde por lo general el estudiantado pertenece al mismo grado y se presentan inconvenientes a la hora de determinar si el papel de tutor se debe de conservar a lo largo de todo el proyecto o no.

A continuación, enumeramos los objetivos concretos que se plantean con la realización de esta propuesta, diferenciando entre estudiantado tutor y tutorizado.

Los objetivos para el estudiantado tutor del grado en Matemática Computacional se centran en mejorar las siguientes competencias presentes en la guía docente de la asignatura:

- Capacidad para comprender y dominar los conceptos fundamentales de matemáticas y aptitud para su aplicación.

- Trabajar intuitiva, geométrica y formalmente con las nociones de álgebra lineal, límite, derivada y derivada parcial.

- Usar programas a nivel avanzado que utilicen cálculo simbólico para manejar los conceptos estudiados en esta materia. 
- Comunicación oral presencial y mediante herramientas TIC.

Objetivos para el estudiantado tutorizado del grado en Química:

- Mejorar las tasas de rendimiento y éxito de la asignatura.

- Mejorar las siguientes competencias presentes en la guía docente de la asignatura:

- Capacidad para manejar las herramientas matemáticas para tratar de una manera rigurosa aquellos aspectos teóricos y prácticos de la Química que lo necesiten.

- Estudio y comprensión de conceptos matemáticos y ser capaz de utilizarlos en los diferentes contextos de la Química.

\section{Desarrollo de la innovación}

\subsection{Diseño del programa same-age tutoring}

Cuando se realiza una tutorización por pares entre estudiantado de un mismo curso del mismo grado, la selección de tutores y tutoras no es siempre una tarea simple. Sin embargo, en nuestro caso, al tratarse de estudiantado de grados distintos, el estudiantado que tutoriza será el de primer curso de Grado en Matemática Computacional, que, en general, se supone que tiene mayor conocimiento y habilidades en la asignatura de matemáticas; y el estudiantado tutorizado será el de primer curso del Grado en Química. Aunque en esta metodología de enseñanza/aprendizaje la ventajas del estudiantado tutorizado parecen más claras, ya que recibe unas 'clases' adicionales a las de su profesorado habitual, tambien existen ventajas claras para el estudiantado tutor ya que es bien sabido que enseñar es aprender dos veces (Topping y Ehly 2001).

En el grado de Matemática Computacional se ofertan 30 plazas de entrada y en el grado en Química 70. Por tanto, en cada grupo habra 3 o 4 personas entre estudiantado tuturizado y tutor.

El profesorado de las asignaturas de Matemáticas I de los dos grados pertenece al área de Geometría y Topología del Departamento de Matemáticas de la Universitat Jaume I de Castelló. En concreto, los autores de este trabajo hemos impartido todas las asignaturas de contenido matemático del grado en Química: Matemáticas I, Matemáticas II y Cálculo Numérico y Estadística; y las asignaturas Matemáticas I, Fundamentos de la Geometría, Geometría Diferencial y Topología, y Aplicaciones de la Geometría Diferencial y la Topología en el grado de Matemática Computacional. Por tanto, tenemos un conocimiento de las asignaturas que se incluyen en el proyecto y también de las necesidades que otras asignaturas van a tener en relación a los contenidos de las asignaturas de Matemáticas I de cada uno de los grados.

Como ya hemos comentado, los contenidos matemáticos de ambas asignaturas son los mismos: álgebra lineal y cálculo diferencial, que se imparten al mismo tiempo a lo largo del primer semestre. Aunque la tipología de la tutorización por pares puede incluir varias dimensiones que dependen del contenido curricular, el año de estudio, tamaño del grupo, características, objetivos...(Topping 1996); por lo que se refiere a la habilidad, aunque muchos proyectos se planifican sobre una base de habilidades cruzadas, en nuestro caso, aun siendo estudiantado del mismo curso, trabajamos sobre la base de que el tutor o tutora tiene un dominio ligeramente superior del contenido matemático, una comprensión más profunda y, con suerte, correcta. 
Planificación, organización y desarrollo del programa. Una vez asignado el estudiantado tutorizado a cada uno de los tutores y tutoras, se analizan los contenidos y los procedimientos de enseñanza que debe aplicar el estudiantado tutor. A continuación, se planifican las actividades que se deben realizar de la forma siguiente:

- Los grupos se deben reunir en cuatro sesiones presenciales (una cada mes), de dos horas aproximadamente de duración, en las que el estudiantado tutor tiene que explicar los conceptos fundamentales de las siguientes materias: Matrices, Espacios vectoriales y Aplicaciones lineales, Cálculo diferencial de una variable y Cálculo diferencial de varias variables.

- Los grupos se tienen que reunir online (semanalmente) para realizar sesiones de tutorización en las que se plantearán la resolución de problemas, con la ayuda de herramientas TIC, y también dudas. Para garantizar el éxito del programa es muy importante la realización de estas sesiones con la mayor frecuencia posible.

En cuanto a las herramientas TIC, hemos distinguido dos tipos según su uso. Por un lado están las herramientas que todo el profesorado y estudiantado de la universidad hemos utilizado de manera acelerada por la situación de pandemia actual, muchas de ellas integradas en el aula virtual de las asignaturas, con el fin de gestionar la comunicación, distribuir materiales docentes y realizar actividades de aprendizaje, trabajo cooperativo y evaluación. Por otro lado, en el campo de las matemáticas, como el proceso de enseñanza-aprendizaje es bastante complicado dado su grado de abstracción, se han propuesto una variedad de caminos metodológicos a fin de lograr mejoras, y un camino destacado es el uso de software educativo matemático. Es decir, hemos incorporado un software (herramienta TIC) con el fin de ayudar al estudiantado a comprender conceptos matemáticos abstractos como el álgebra lineal y las derivadas, así como sus aplicaciones e interpretaciones.

Existen muchos programas de cálculo simbólico como Mathematica, Maple, Matlab, Derive... También existen páginas web como WolframAlpha, e incluso apps para móviles como Geogebra, que pueden usarse como software matemático educativo (Pineda, Hernández y Avendaño 2020), (Gil, Calvo y Gil 2011).

En este proyecto hemos utilizado el programa Mathematica, ya que también se utiliza en la asignatura Nuevas Tecnologías de la Información y Computación aplicadas a la Química, que se cursa en el segundo semestre del primer curso del grado en Química.

En las horas de tutoría presenciales de las asignaturas de los dos grados, el profesorado revisamos el contenido de los conceptos teóricos que los grupos trabajarán en las sesiones presenciales, así como las dudas y la resolución de problemas con la ayuda de Mathematica. En el caso del estudiantado del grado en Matemática Computacional la participación en el proyecto cuenta dentro del apartado de evaluación de las competencias adquiridas en los seminarios: máximo 2 puntos sobre 10. Para el estudiantado del grado en Química, la participación en el programa forma parte de la evaluación de resolución de problemas en el aula y colección de problemas resueltos: máximo 2 puntos sobre 10 .

En la organización y preparación de las tutorías presenciales de las asignaturas el profesorado nos coordinamos para fijar los problemas a realizar por los estudiantes y mostrar ejemplos del uso de Mathematica en la resolución de los mismos. 


\subsection{Dinámica de las clases presenciales y en línea}

En el proyecto educativo utilizamos el aprendizaje semipresencial (blended learning) y, por tanto, se combina el trabajo presencial (en espacios docentes seguros de la universidad) con el trabajo en línea a través de medios digitales. Cada vez que se explica por parte del profesorado un concepto de álgebra lineal o cálculo diferencial, el estudiantado tutor tiene que comprender el concepto, demostrar los resultados pertinentes, realizar un esquema conceptual y, después, en la clase de tutorización presencial mensual tiene que explicar al estudiantado tutorizado los conceptos correspondientes, sin entrar en demostraciones formales. A su vez, en las sesiones en línea, se analizan los ejemplos preparados que ilustran los conceptos teóricos, se visualizan y se resuelven problemas.

En la Tabla 1 se detallan los aspectos a desarrollar por el estudiantato tutor y tutorizado, con está dinámica de clases, para el concepto de derivada de una función.

Tabla 1: Actividades a desarrollar en el concepto de derivada

Estudiantado tutor

Clase presencial Concepto de derivada. Recta tangente. Interpretación geométrica de la ecuación tangente.

Ecuación de la recta tangente por medio de la derivada.

Clases en línea

Utilizar el programa de cálculo simbólico para explicar el concepto de derivada y de recta tangente.

Construir gráficas de funciones e intersecciones de planos y superficies.

Actitudes
Estudiantado tutorizado

Cálculo de derivadas.

Cálculo de la pendiente de la recta tangente. Determinación de la ecuación de la recta tangente.

Visualizar el concepto de derivada y de recta tangente.

Utilizar el programa de cálculo simbólico para resolver derivadas.

Organize su tiempo y trabajo.

Considere fundamental el concepto de derivadas para estudiar fenómenos físico-químicos. Utilice software informático como apoyo en la resolución de derivadas.

\section{Resultados}

El presente proyecto se trata de una experiencia piloto que se plantea por primera vez entre el estudiantado de primer curso de los grados en Matemática Computacional y en Química.

Para analizar los resultados obtenidos en el proyecto y ver si se consiguen los objetivos concretos planteados inicialmente, nos basamos en dos variables: los resultados de la evaluación continua y de la evaluación final de las dos asignaturas y la realización de una encuenta al estudiantado. 
La primera variable en el caso del estudiantado tutor no aporta demasiada información. Hay que tener en cuenta que los 30 estudiantes del grado en Matemática Computacional tienen resultados brillantes en el bachillerato científico, les gustan las matemáticas y esta asignatura contiene un elevado porcentaje de contenidos estudiados en segundo curso de bachillerato. Además, este estudiantado participa de manera activa en las sesiones de evaluación continua y obtiene buenos resultados en la evaluación final. Por tanto, no se espera un cambio significativo en la consecución de los objetivos a partir de esta variable.

Sin embargo, en el caso del estudiantado tutorizado esta variable sí resulta de interés. En cursos anteriores sobre un 25 por ciento del estudiantado abandonaba las sesiones de evaluación continua sobre resolución de problemas, este proyecto tiene impacto sobre este abandono y, por tanto, teniendo en cuenta que el sistema de evaluación de las asignatura no cambia, el objetivo planteado inicialmente como mejora de las tasas de rendimiento y de éxito de la asignatura se debe cumplir.

La Tabla 2 incluye la encuesta para el estudiantado una vez finalizado el programa sobre esta nueva propuesta metodológica para la enseñanza de las matemáticas con Mathematica y con la tutorización por pares.

Tabla 2: Encuesta para el estudiantado participante en el programa

Respuesta: Sí, No, No sé Estudiantado tutor $\quad$ Estudiantado tutorizado

$\begin{array}{lll}\text { Primera pregunta. } & \text { El programa me ha servido para compren- } & \text { El programa me ha servido para mejorar } \\ \text { der y dominar los conceptos fundamenta- } & \text { mi comprensión de conceptos matemáti- } \\ \text { les de la asignatura Matemáticas I y me- } & \text { cos y ser capaz de utilizarlos en los dife- } \\ \text { jorar mi aptitud para su aplicación. } & \text { rentes contextos de la Química. }\end{array}$

Segunda pregunta.

El programa me ha servido para usar programas de cálculo simbólico para manejar los conceptos estudiados en esta materia.

Tercera pregunta.

El programa me ha servido para comunicar mejor resultados de matemáticas.
El uso de herramientas TIC en el programa ha hecho más entretenida la tarea de resolver problemas de matemáticas.

El programa me ha servido para motivarme a la hora de seguir las tareas de evaluación continua.

Ampliaría el programa a otras asignaturas del grado.
Cuarta pregunta.

Ampliaría el programa a otras asignaturas del grado.

De las respuestas de la encuesta los resultados esperables son los siguientes:

- Al estudiantado del Grado en Matemática Computacional el programa le ayuda a comprender mejor algunos conceptos de la asignatura. Para el estudiantado del grado en Química el programa le sirve para comprender y aplicar los conceptos matemáticos de la asignatura. 
- Para el estudiantado del grado en Química el uso de los recursos tecnológicos hace más "entretenida" la materia, mientras que para la mayoría del estudiantado del Grado en Matemática Computacional le sirve como introducción a otros lenguages de programación.

- Al estudiantado del Grado en Matemática Computacional el propgrama le sirve para mejorar su capacidad de comunicación oral de las matemáticas. Al estudiantado del grado en Química las tutorías le ayudan a mejorar su motivación por la materia.

- Al estudiantado de ambos grados le interesa que se siga empleando la metodología en otras asignaturas del grado; de hecho, existen dos asignaturas de Matemáticas II de segundo semestre, con contenidos de Cálculo Integral y Ecuaciones Diferenciales.

\section{Conclusiones}

El estudiantado de primer curso y primer semestre de los grados de Matemática Computacional y Química de la Universitat Jaume I de Castelló tiene que cursar la asignatura Matemáticas I, cuyos contenidos son, en ambos casos, Álgebra Lineal y Cálculo Diferencial. Ahora bien, la manera de enfocar la enseñanza de estos contenidos en los dos grados es distinta, ya que el interés con el que el estudiantado aborda esta asignatura y los objetivos que se pretenden alcanzar en cada caso son muy distintos. El estudiantado del grado en Matemática Computacional no sólo debe entender y aplicar los conceptos, sino que debe razonar y demostrar las afirmaciones; sin embargo, para el estudiantado del grado en Química las matemáticas son una herramienta (abstracta y compleja) que debe entender para aplicarla en la resolución de problemas físico-químicos. Esta combinación hace, a nuestro entender, perfecta la introducción de la tutoría por pares entre este estudiantado de la misma edad, de manera que el estudiantado tutor de Matemática Computacional aprenda enseñando los conceptos de matemáticas, con la ayuda de un programa de cálculo simbólico, y para el estudiantado de química, el hecho de realizar sesiones presenciales y en línea con estudiantado de su misma edad le ayude a perder 'el miedo' a las matemáticas.

El proyecto piloto propuesto en este trabajo junto con los resultados previstos, nos llevan a concluir que la introducción de esta metodología same-age tutoring, utilizando el aprendizaje semipresencial (blended learning) y el uso de las herramientas TIC en el doble sentido de gestionar la comunicación, distribuir materiales docentes y realizar trabajo cooperativo, e introducir un programa de cálculo simbólico, resulta sin duda provechosa tanto para el estudiantado tutor como para el tutorizado.

El análisis de las tasas de abandono y rendimiento académico y las respuestas del estudiantado a la encuesta planteada al final del proyecto, abren la vía para que el programa ayude en la comprensión y dominio de los conceptos matemáticos, sirva para que el estudiantado del grado en Química se motive en el estudio de la asignatura de matemáticas y fomente la mejora comunicativa del estudiantado tutor.

Sin embargo, no resulta fácil ampliar el proyecto a otro tipo de asignaturas ya que no es fácil encontrar dos asignaturas con contenidos prácticamente iguales y que se impartan en grados distintos como son Matemática Computacional y Química. 


\section{Referencias bibliográficas}

Alegre, F. y col. (2020). "Academic Achievement and Peer Tutoring in Mathematics: A Comparison Between Primary and Secondary Education". En: SAGE Open, April-June, págs. 1-92.

Alzate-Medina, G.M. y L.B. Peña-Borrero (2010). "La tutoría entre iguales: una modalidad para el desarrollo de la escritura en la educación superior". En: Universitas Psychologica 9(1), págs. 123-138.

Gil, L.G., I. Calvo e Y.B. Gil (2011). El Uso De TIC Como Medio Para La Enseñanza Del Álgebra Lineal. Recife, Brasil.

Grisales-Aguirre, A.M. (2018). "Uso de recursos TIC en la enseñanza de las matemáticas: retos y perspectivas". En: Entramado 14(2), págs. 198-241.

Pineda, W.B., C.A. Hernández y W.R. Avendaño (2020). "Propuesta didáctica para el aprendizaje de la derivada con Derive". En: Praxis 83 Saber 11(26), págs. 1-19.

Real-Pérez, M. (2011). Las TIC en el proceso de ensenanza y aprendizaje de las matematicas. Jornadas de Innovacion docente. Facultad de Matematicas. Universidad de Sevila.

Robinson, D.B., J.W. Schofield y K.L. Steers-Wentzell (2005). "Peer and Cross-Age Tutoring in Math: Outcomes and Their Design Implications". En: Educational Psychology Review 17(4), págs. 327-362.

Santaló, L.A. (1986). La matemática en la educación. Editorial Docencia. Argentina.

Topping, K.J. (1996). "The effectiveness of peer tutoring in further and higher education: A typology and review of the literature". En: Higher Education 32(3), págs. 321-345.

Topping, K.J. y S.W. Ehly (2001). "Peer assisted learning: A framework for consultation". En: Journal of Educational and Psychological Consultation 12(2), págs. 113-132. 